\title{
Coronary Artery Graft
}

National Cancer Institute

\section{Source}

National Cancer Institute. Coronary Artery Graft. NCI Thesaurus. Code C100064.

A vessel segment attached to a coronary artery to facilitate myocardial perfusion. (ACC) 